\title{
Femme, seule et venue d'ailleurs. Trois atouts d'un ethnomusicologue au Turkestan chinois
}

\author{
A woman, a woman alone and an outsider. Three assets of an ethnomusicologist \\ in Chinese Turkestan
}

\section{Sabine Trebinjac}

\section{OpenEdition}

\section{Journals}

Édition électronique

URL : http://journals.openedition.org/ethnomusicologie/1189

ISSN : 2235-7688

\section{Éditeur}

ADEM - Ateliers d'ethnomusicologie

\section{Édition imprimée}

Date de publication : 31 décembre 1995

Pagination : 59-68

ISBN : 2-8257-0537-3

ISSN : 1662-372X

\section{Référence électronique}

Sabine Trebinjac, "Femme, seule et venue d'ailleurs. Trois atouts d'un ethnomusicologue au

Turkestan chinois », Cahiers d'ethnomusicologie [En ligne], 8 | 1995, mis en ligne le 04 janvier 2012, consulté le 23 avril 2019. URL : http://journals.openedition.org/ethnomusicologie/1189

Ce document a été généré automatiquement le 23 avril 2019

Tous droits réservés 


\title{
Femme, seule et venue d'ailleurs. Trois atouts d'un ethnomusicologue au Turkestan chinois
}

\author{
A woman, a woman alone and an outsider. Three assets of an ethnomusicologist \\ in Chinese Turkestan
}

Sabine Trebinjac

1 Le Turkestan chinois ou, pour user de son appellation contemporaine, la Région autonome ouïgoure du Xinjiang, est situé à l'extrême nord-ouest de la République Populaire de Chine et partage ses frontières avec sept États voisins. D'une superficie triple de celle de la France (plus de 1,6 millions de $\mathrm{km}^{2}$ ), le Xinjiang a la particularité d'être une région bordée d'importants systèmes montagneux qui laissent une large place aux déserts ( $22 \%$ de la superficie totale); elle connait en outre un climat des plus continentaux qui offre une amplitude thermique peu fréquente (jusqu'à $40^{\circ} \mathrm{C}$ ). Autrement dit, les caractéristiques physiques du Turkestan chinois ne sont pas pour rendre la région fort avenante.

2 Peuplé de plus de quatorze millions d'habitants, le Xinjiang se présente comme une sorte de mosaïque ethnique puisque dix-sept ethnies différentes s'y côtoient. Ces dernières peuvent être regroupées, ce qui nous conduit à considérer grosso modo trois grandes familles se répartissant de la manière suivante: les Ouïgours qui, bien qu'encore majoritaires, ne représentent plus qu'une petite moitié de l'ensemble de la population de la région; les Hans (auxquels on assimile ici les Huis, Hans musulmans), dont le nombre de ressortissants ne cesse de croître, constituent pour l'heure le deuxième groupe quantitativement le plus important, et enfin les populations turco-mongoles, autres que les Ouïgours, forment le troisième groupe. Or, la cohabitation de ces diverses ethnies, qui pour certaines n'ont que la citoyenneté chinoise en commun, ne va pas sans heurts. Au meilleur des cas, on se côtoie, mais sans se fréquenter et, au pire, on s'affronte dans des conflits qui sont quelquefois violents. Autant dire que les données humaines, loin 
d'atténuer les rigueurs imputables aux éléments naturels, contribuent au contraire à rendre le climat social tendu, comme porteur d'une trop grande intensité électrique.

Tel est, brossé à gros traits, le décor de ce coin de Chine où pléthore d'« ethnomusicologues » chinois sont amenés depuis 1950 à «faire du terrain», notamment au cours des années d'enquêtes ethnologiques dirigées par l'anthropologue Fei Xiao-tong qui devait aboutir en 1955 à la proclamation de la Région autonome ouïgoure. On l'aura compris, la venue de ces «scientifiques» - Hans pour la plupart n'est pas motivée par un engouement pour la région, ni pour les musiques qui y sont jouées, et moins encore pour les musiciens. En fait, seules sont à invoquer des raisons politiques. Autrement dit, ils n'y viennent pas par goût, mais par devoir. Afin de bien comprendre cette logique a priori étrange, il nous faut revenir sur ce qu'est un ethnomusicologue chinois.

4 C'est un fonctionnaire qui collecte les musiques de la République. Mais loin s'en faut qu'ils soient tous des Brăiloiu chinois car qui dit collecte ne signifie pas compilations de musiques recueillies. En effet, la collecte en Chine suppose aussi la «mise en ordre", zhengli, le façonnage, jia gong, la transformation, gai bian, et l'écriture, chuang zuo, ou plutôt la réécriture, des musiques. Pour ce faire, un long processus centralisé et hiérarchisé est mis en œuvre. Disons pour résumer ${ }^{1}$ que, calquée sur l'organisation territoriale, une collecte se déroule en trois phases successives, impliquant des fonctionnaires de niveau d'abord national, puis provincial et enfin local. Un centre de décision donne l'ordre de collecte à ses subordonnés provinciaux, qui eux-mêmes le transmettent à leurs représentants locaux. Ces derniers exécutent alors le travail de compilation sur le terrain et en envoient le fruit à leurs supérieurs provinciaux qui, comme précédemment mais en sens inverse, le transmettent à leur tour à la direction nationale. Cette chaîne tripartite ne répond manifestement pas à un souci d'efficacité qui voudrait améliorer les moyens et les délais de communication. En fait, elle permet non seulement de surveiller ce que fait le subalterne (nous sommes, ne l'oublions pas, dans un État totalitaire), mais aussi d'aller au devant des souhaits émanant des instances hiérarchiquement supérieures (c'est ce point, révélant une coopération de la base avec le sommet, qui constitue une des spécifités de la Chine en tant qu'État totalitaire). Car, pratiquemment, des "nettoyages", des "améliorations " successives jalonnent cette lente ascension des mélodies recueillies : à chacune des étapes, y compris la première, les fonctionnaires « arrangent » ce qui leur a été transmis. Bien évidemment, et parce qu'il s'agit d'une organisation hiérarchique, c'est au sommet de la pyramide que les transformations sont les plus importantes.

5 La collecte musicale est alors réalisée... à moitié. En effet, commence seulement pour ces mélodies « revues et corrigées » le chemin du retour, puisqu'elles sont réexpédiées sur leur terre d'origine par la même voie qu'avait antérieurement suivi l'ordre de collecte. Ce n'est que lorsque les formes réécrites des mélodies remplacent au niveau local les versions originales que la « collecte » est enfin achevée.

6 On aura donc compris qu'une collecte musicale est, en Chine, l'œuvre non pas d'un petit nombre d'ethnomusicologues mais d'une armée de fonctionnaires; elle n'est pas confinée dans les tiroirs d'un quelconque Institut de recherches, ni réservée à l'usage de rares spécialistes, mais elle est destinée à la nation toute entière.

7 Telles sont les tâches que doivent remplir les ethnomusicologues chinois. 
Fig. 1 : Un fonctionnaire de la musique (deuxième depuis la gauche), un fonctionnaire des Affaires militaires (troisième depuis la gauche) et le chauffeur de la Jeep (quatrième depuis la gauche, à l'arrière) rendant visite à une famille tadjike de paysans-musiciens. District de Tašqorgan, mai 1988.

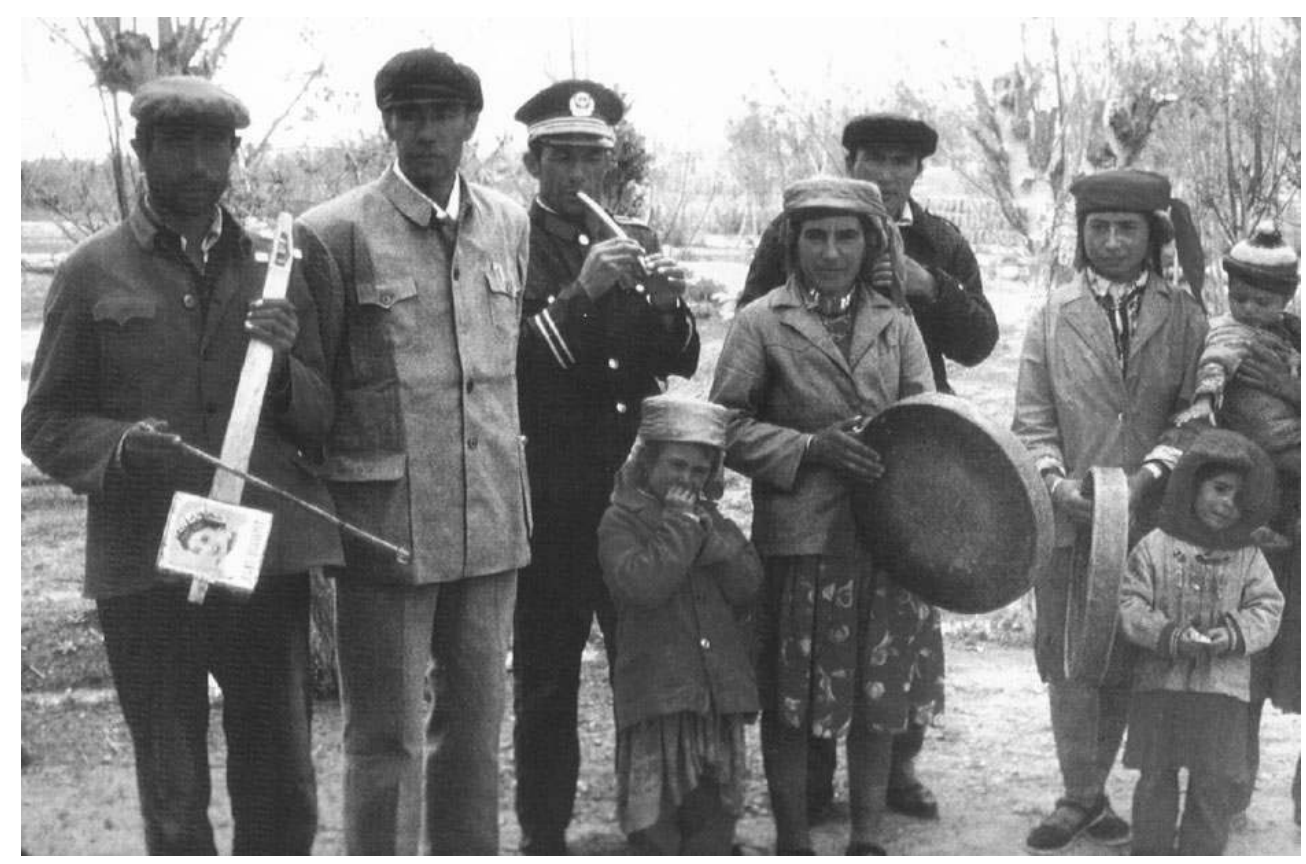

Photo : Sabine Trebinjac.

\section{Deux types d'ethnomusicologues chinois}

Voyons, à présent, comment ces fonctionnaires procèdent pratiquement sur le terrain et, plus précisément, dans la région du Xinjiang. Comme partout et de même que pour de nombreuses autres disciplines, on peut estimer qu'il existe autant de pratiques que de praticiens. Cependant, l'occurrence de certains traits et l'observation répétée que j'ai été amenée à en faire sur place me permettent d'envisager des types d'enquêteurs auxquels sont liées des façons de faire ethnomusicologiques.

Les ethnomusicologues chinois se rendant en terrain ouïgour sont soit Han soit Ouïgour ; tous se déplacent au moins à deux (que le deuxième soit interprète, technicien ou chauffeur) ; à ma connaissance, tous appartiennent à la gent masculine ; ils relèvent en grande partie de l'échelon local, pour une plus petite part de l'échelon provincial et de façon tout-à-fait exceptionnelle des hautes instances pékinoises. Ces premières caractéristiques ainsi établies déterminent forcément des types d'ethnomusicologues. Car un Ouïgour, fonctionnaire dans une " unité de travail» locale, n'agit ni ne réagit de la même façon qu'un de ses collègues han pékinois, pour lequel parcourir le Xinjiang, c'est voyager à l'étranger. Mon propos n'étant pas ici de décrire en détails la typologie des ethnomusicologues chinois; je me bornerai pour l'heure à ne traiter que de deux types qui s'inscrivent à l'opposé l'un de l'autre. Ces exemples permettront au lecteur d'anticiper la suite et de percevoir pourquoi, lorsque j'ai décidé de m'intéresser à la musique ouïgoure, je ne pouvais être appréhendée que comme un personnage atypique. 
10 Prenons d'abord le cas d'un Ouïgour, fonctionnaire dans une « unité de travail » locale. Etant donnée la tension ethnique qui règne au Xinjiang, c'est généralement lui et non son collègue han qui est chargé du travail de collecte. Pour ce faire, il sillonne les campagnes en Jipu che ${ }^{2}$, accompagné du chauffeur de service, muni de son carnet et d'un appareil photographique. La possibilité d'emporter le magnétophone de son unité de travail est bien mince puisqu'il n'existe qu'une seule machine de ce genre et que cela suppose en outre qu'elle soit en état de marche à l'heure du départ et que les crédits permettent l'achat de piles en quantité suffisante. Enfin, s'il a bien géré son budget ${ }^{3}$, il glissera dans ses poches quelque argent. Arrivé dans un petit village, le collecteur prend contact avec la «Station de la Culture » du coin qui organise pour lui les rencontres avec les musiciens. Il établit alors leur fiche biographique, transcrit leurs chansons, les photographie ainsi que leurs instruments, dont il aura dressé un relevé organologique très détaillé, etc.

11 J'ai eu l'occasion de côtoyer un de ces collecteurs locaux ouïgours quotidiennement et pendant une longue période. Lui-même musicien, il accomplissait son travail lentement mais avec soin. En outre, il se sentait investi d'une mission de sauvegarde de la culture populaire ouïgoure. Au fil des années, il avait ainsi accumulé des documents sur les musiciens et les musiques de toute la partie sud du Xinjiang. Mais il était toujours réticent à communiquer ses archives à ses supérieurs. Cette attitude de méfiance est d'ailleurs tout à fait comparable avec celle des vieux musiciens. En effet, tout comme ce collecteur cache dans ses tiroirs le résultat de son travail, les musiciens trompent les enquêteurs qu'ils ne connaissent pas et tout particulièrement ceux qui viennent de la capitale régionale. Ainsi, ce vieux musicien qui, avec un sourire malicieux, me déclara: «Je connais dix-sept muqam et je te les apprendrai, mais n'en dis rien car je n'en ai déclaré que quinze aux enquêteurs d'Urumči », ou cette femme septuagénaire qui après avoir chanté et joué du tanbur pendant deux jours me dit, en riant: "Surtout ne fais pas écouter tes enregistrements aux spécialistes hans, parce que voilà déjà cinq ans que je fais semblant d'être handicapée du bras et aphone quand ils viennent enquêter ». Les mensonges sont toujours présentés comme une petite victoire des musiciens vis-à-vis de la grande machine administrative. Cette revanche est motivée par deux facteurs dépendants. Premièrement, les musiciens sont déconsidérés en Chine. Au Xinjiang, la plupart d'entre eux ne doivent leur subsistance qu'à leur petit champ ou à quelques moutons et ne peuvent compter sur leur pratique musicale pour subvenir à leurs besoins. Ils sont donc envieux envers les musiciens devenus fonctionnaires, auxquels ils ne tiennent pas à communiquer leur savoir. Deuxièmement, les vieux musiciens reconnaissent quelquefois en écoutant la radio leur propre chanson qui a été « arrangée » par un musicien collecteur qui est alors présenté comme l'auteur de la pièce. Ils se sentent donc dépouillés de leur bien et nourrissent une forte rancune envers le faussaire. C'est pourquoi ils cachent leur savoir et préfèrent disparaitre avec, même si cela doit être fatal à la musique ouïgoure. Dans certains cas, cependant, une relation privilégiée peut aussi s'instaurer entre le musicien et le collecteur local. En effet, des liens familiaux ou un réseau amical peuvent intervenir entre le fonctionnaire et le musicien, ce qui favorise grandement le travail de collecte. Et c'est là d'ailleurs un point dont ont été conscients les dirigeants. Ils ont très vite compris que, pour lutter contre les réticences des vieux musiciens à livrer leur répertoire, il fallait mettre à profit le tissu social qui peut entourer le collecteur et qui lui permet de mener à bien son travail. À cette cohésion sociale s'ajoute également une forte solidarité de groupe. Le musicien qui est «monté » à la grande ville et a été promu fonctionnaire a incontestablement réussi. On lui doit alors 
non seulement le respect, mais encore toutes sortes d'aide qui peuvent lui faciliter la tâche, car, hormis sa promotion personnelle, il favorise aussi la montée de son groupe d'origine dans la hiérarchie sociale.

Fig. 2 : Le grand maître du muqam ouïgour, Turdi Ahon, entouré d'ethnomusicologues chinois, hans en majorité. In : Säypidin Äzizi, Uyǵur muqami togrisida (Beijing, millätlär näšriyati, 1992, non paginé.

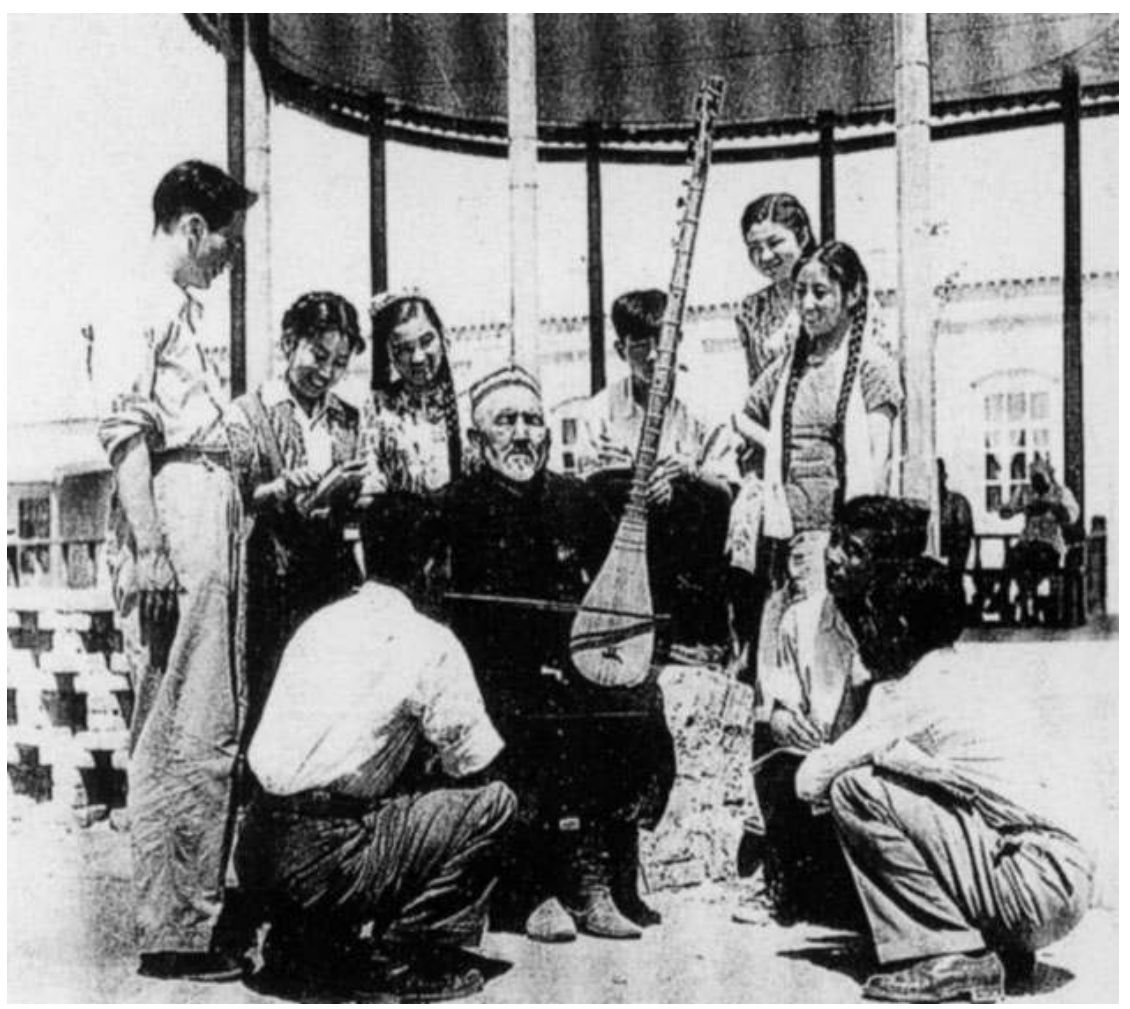

Envisageons à présent le cas de l'ethnomusicologue han qui débarque de Pékin. Comme il a été dit précédemment, rares sont les fonctionnaires de rang national à se rendre euxmêmes sur place. Comme leurs collègues régionaux, ils ne prennent généralement pas la peine de "descendre " au niveau local pour effectuer les collectes sur le terrain et se contentent, la plupart du temps, d'envoyer à leurs subordonnés l'ordre de collecter des musiques. Il est intéressant de noter que ceux qui, de nos jours, « voyagent » au Xinjiang y ont déjà séjourné plus ou moins longtemps par le passé dans des circonstances tout à fait particulières. En effet, la majorité d'entre eux n'ont dû qu'à des conjonctures politiques ou militaires le fait d'avoir été « déplacés » au Xinjiang, que c'eût été en tant que militants de la "politique de sauvegarde des arts des minorités » édictée peu après 1949, ou que ce fut en tant que soldats de l'Armée de Libération ou encore, comme victimes ou descendants de victimes des Gardes Rouges lors de la Révolution Culturelle. Cette brève évocation des diverses raisons de leur première arrivée au Xinjiang mérite d'être signalée car elle n'est pas sans incidence sur l'attitude qu'adoptent aujourd'hui encore ces anciens «exilés » de l'intérieur vis-à-vis de l'environnement ouïgour, tant humain que naturel. L'ethnomusicologue pékinois, aussi familier soit-il avec le terrain ouïgour, ne s'exprime pas avec les musiciens ouïgours dans leur langue; il se fait accompagner par un interprète. Ses tournées sur place sont très courtes, quelques jours lui suffisent pour recueillir ce qu'il est venu y chercher. En général, les choses se passent de la manière suivante : avant son arrivée, le bureau de la Culture a été averti de sa visite et le chef dudit bureau a donc eu tout loisir de préparer le terrain en dépêchant ses 
adjoints à la recherche des meilleurs et des plus vieux musiciens. Lorsque la saison appelle plus aux travaux des champs qu'au jeu musical, il n'hésite pas à allouer une prime aux musiciens qui, ne pouvant la refuser, s'engagent ainsi à venir. Au jour et au lieu dits (c'est-à-dire soit dans les bâtiments réservés à la troupe locale de chants et de danses, soit dans la chambre d'hôtel dans lequel est descendu le fonctionnaire), la rencontre a lieu entre le collecteur et les musiciens. Celle-ci dure en moyenne deux heures et consiste en général en une séance d'enregistrements, une autre de photographies et un entretien conduit sur le mode de l'interview dirigée. J'ai été amenée à constater que très souvent tous les éléments de réponse attendus par l'ethnomusicologue de rang national - voire même par celui d'échelon provincial - figuraient dans les questions; les musiciens donnent alors plus l'impression d'obtempérer aux desiderata d'un bureaucrate que de livrer quelque information que ce soit sur leur tradition musicale. Après ces quelques heures de travail, le collecteur s'en retourne non sans avoir promis de tout faire pour mettre à profit «le savoir que les vieux lui ont transmis ». Autant dire que les enquêtes conduites par ce type d'ethnomusicologues sont rondement menées.

\section{Une intrusion atypique}

13 A travers ces petits portraits d'ethnomusicologues chinois brièvement esquissés, voici présenté ce à quoi les musiciens ouïgours sont habitués. Le terme d'habitude n'est pas trop fort car on remarque que, de même que le travail et les façons de faire des enquêteurs sont coutumiers, de même le comportement des enquêtés paraît être totalement conditionné par les collecteurs chinois: à question de fonctionnaire, réponse bureaucratique. Autrement dit, cela signifie que, vis-à-vis de l'espèce des ethnomusicologues, les musiciens ouigours nourrissent a priori une inimitié qui peut tendre vers une hostilité plus ou moins sourde.

C'est donc dans ce contexte que je décidai de mener des recherches ethnomusicologiques au cours de l'année 1988-1989. Qu'il me soit tout d'abord permis de signaler, en réplique aux caractéristiques des collègues chinois précédemment relevées, quelques traits personnels : ma venue au Xinjiang ne résultait pas d'un ordre politique émanant d'une quelconque instance hiérarchique supérieure, mais était motivée par des préoccupations sociologiques et musicales qui m'étaient propres; je voyageais seule, en transport en commun et à pied ; je conversais avec les Ouïgours en ouïgour ; je n'étais ni fonctionnaire, ni chinoise; enfin, j'appartenais au genre féminin. C'est-à-dire que, malgré mes dires d'être une ethnomusicologue, je différais par trop du cliché pour ne pas être placée horscatégorie. Or, cette situation, qui ailleurs aurait pu s'avérer malheureuse, fut fort profitable puisque les principaux traits «atypiques» que ma personne combinait devinrent autant d'atouts.

Pendant au moins les trois premiers mois de mon séjour, j'entretins avec les instances officielles locales des relations empreintes d'une mutuelle suspicion: elles considéraient que ma curiosité à l'endroit de la musique ouiggoure était un prétexte fallacieux et restaient persuadées que mon établissement dans ces régions frontalières s'inscrivait dans une opération organisée par un quelconque service secret (probablement français); pour ma part, et sans doute parce que j'étais lasse de leur surveillance quotidienne, je ne voyais en elles qu'une force d'opposition, dont le rôle se résumait à refuser toutes mes demandes de permis de circulation intérieure. Cette bonne guerre ne devait pas durer car, de leur côté, force leur fut de constater qu'aucun de mes agissements ne contrevenait 
à leurs règles et, personnellement, ma connaissance de la langue et du savoir-être ouïgours m'autorisèrent à me dispenser de leurs autorisations de circuler. Mais l'épisode fut néanmoins suffisamment long et de nature assez cocasse pour être connu de beaucoup et pour m'attirer le regard complaisant des musiciens. Car j'étais devenue une ethnomusicologue qui, pour être en mesure de les rencontrer, osait tenir tête aux autorités et qui jouait à être ouïgoure pour être libre de ses mouvements. Telle est une des raisons pour lesquelles les relations que je nouais avec les musiciens furent toujours empreintes d'un sentiment de confiance et de bienveillance quelquefois tutélaire. Mais il en est d'autres.

Etre une étrangère est aussi un avantage, celui de n'être pas han. Cela implique qu'il était possible de me conter sans crainte des confidences ou un savoir qui est considéré comme n'appartenant qu'aux seuls Ouïgours, c'est-à-dire a priori intransmissible aux Hans. Et puis, être une étrangère ouvre encore une autre possibilité, insoupçonnée jusqu'alors : celle de pouvoir quelquefois être considérée comme asexuée. En effet, c'est bien ainsi, et grâce à cette qualité, qu'il m'a été possible de me joindre à des activités exclusivement masculines telles que, par exemple, les séances soufies de zekr. Parée d'un foulard afin de ne pas heurter la sensibilité des participants habituels, ma présence était tolérée parce que mon statut d'étrangère permettait que je ne sois plus une femme et cependant pas un homme. L'espace d'un moment, je perdais à leurs yeux toute identité sexuelle, je n'étais plus qu'une personne étrangère... Il est intéressant de noter que cette faculté de mutation est, par contre, refusée aux hommes. En effet, il découlait des discussions portant sur ce sujet, tant avec des hommes que des femmes ouïgours, qu'un homme même étranger est voué à rester d'abord un homme. Où que ce soit, et plus encore dans un milieu exclusivement féminin, il n'est pas possible de l'accepter au titre unique d'étranger. Cette différence de traitement est, je pense, à imputer à l'image que les Ouïgours se font de l'homme. Celui-ci est d'abord représentant du «sexe fort " et doit toujours afficher sa virilité ; chercher à la cacher ou « faire la femme ${ }^{4}$ sont des attitudes honteuses, indignes d'un « vrai » homme.

Paradoxalement, il était quelquefois plus difficile de se faire accepter par les femmes en tant que femme. Peu à peu, je compris cette réticence, a priori suprenante, comme étant une manifestation de crainte à l'encontre de leur entourage masculin. En effet, le fait qu'elles aient des activités de groupe à l'écart des regards masculins n'est pas toujours pour plaire aux hommes. C'est ce que je devais comprendre au cours d'un zekr féminin lorsque, suite à un malaise que l'on pouvait facilement déceler dans l'ambiance, les participantes, interrompant subitement la séance, m'invitèrent à expliquer les raisons de ma présence parmi elles. Or, autorisation m'en avait été donnée la veille au soir par la maîtresse de cérémonie elle-même, au terme de plusieurs heures de discussions et d'explications qui s'achevèrent par cette phrase: «Tu peux venir, puisque tu es une femme ». Puis, le zekr terminé, la bonne centaine de femmes réunies émirent vivement le souhait que je ne fasse jamais écouter à des hommes les enregistrements effectués. Je promis et ne devais comprendre que plus tard la raison de ce vœu : nombre d'entre elles participent à ces séances hebdomadaires à l'insu des hommes de leur parenté, aussi craignaient-elles d'être reconnues si jamais l'un d'entre eux venait à entendre mes bandes...

18 S'il est donc bon d'être femme parmi les femmes, il peut être parfois difficile de l'être parmi les hommes, pour des raisons que l'on devine aisément, surtout là où la force virile est valorisée et lorsque le rival est absent. Pourtant, et malgré quelques mésaventures 
finalement sans importance, le fait d'être non accompagnée s'est avéré être un autre atout. On a déjà souligné l'habitude contractée par les ethnomusicologues chinois de se déplacer sur le terrain au moins à deux. En outre, dans cette terre islamisée - même si les pratiques musulmanes y sont fortement « ouïgourisées » - l'indépendance et l'initiative ne sont pas des qualités qui sont facilement accordées aux femmes. Aussi, le fait d'être femme, seule et jeune est en soi extra-ordinaire. En conséquence, la réaction que la plupart de mes interlocuteurs manifestaient à mon égard, loin de me repousser, était de « m'adopter » qui, comme leur fille, qui, comme leur sœur. On doit ici noter que ce type "d'adoption spontanée » n'est pas propre aux Ouïgours et est assez commun dans l'ensemble de la Chine où on laisse rarement seule une personne rencontrée : il est plus agréable de marcher en compagnie; quand il y a à manger pour trois, il y en a pour quatre ; dans un foyer il est facile d'improviser une couche supplémentaire, etc.

Telles sont les raisons qui font qu'être femme, ne pas être han, et être seule sont autant d'« attributs» bénéfiques pour une ethnomusicologue française au Turkestan chinois. Cependant, on peut être étonné que les vieux musiciens m'aient livré leurs confidences. Après tout, n'étais-je pas, moi aussi et malgré mes différences, assimilable à un collecteur? Les craintes de certains, très rares il est vrai, selon lesquelles mes enregistrements étaient non seulement assimilables à un vol, mais pouvaient aussi me permettre de m'en déclarer l'auteur en France, loin de leurs oreilles, n'étaient-elles pas fondées? Sans doute les explications que je fournissais sur mon travail ont-elles été convaincantes ; je n'ai en tout cas jamais rencontré la moindre difficulté pour enregistrer et encore moins pour m'entretenir avec les musiciens.

Deux ans plus tard, en 1990, je suis retournée en Chine en apportant le disque consacré à la musique ouïgoure (Trebinjac et During 1990) produit entre temps. Tous mes interlocuteurs connus par le passé, qu'ils fussent musiciens ou fonctionnaires de la musique, en furent désappointés : j'avais publié un disque de leurs musiques sans les avoir réécrites ; c'est donc la matière brute musicale que j'ai soumis au public français. Comble d'étonnement, un éditeur avait publié ça, que des gens étaient censés acheter ! Et de conclure qu'ethnomusicologues ou pas, les Français sont vraiment trop bizarres... !

\section{BIBLIOGRAPHIE}

TREBINJAC Sabine, 1996, Musique ouïgoure et collectes musicales en Chine, thèse de doctorat en ethnologie et sociologie comparative, Paris X-Nanterre, 1993 (version éditée par la Société d'ethnologie, Nanterre, à paraître).

TREBINJAC Sabine et Jean DURING, 1990, Turkestan chinois. Musique savante des muqam (vol. I) Tradition populaire des Oügours (vol. II), Radio France-Ocora \& AIMP / Musée d'Ethnographie de Genève, 1990. 


\section{NOTES}

1. Les collectes musicales en Chine ont été au centre d'un important travail de recherche réalisé précédemment. C'est pourquoi je ne reviendrai pas en détails sur leur déroulement et me permets de renvoyer au dit travail (Trebinjac 1996).

2. Il s'agit de la version chinoise de la Jeep américaine.

3. En 1988, le budget annuel des services locaux pour le travail de collecte s'élevait à 500 yuans un yuan valant à l'époque deux francs français au cours officiel.

4. Cette expression ouigoure recouvre quantités d'occupations ou d'attitudes qui sont considérées ne relever que du domaine des femmes et qui, par conséquent, sont interdites aux hommes ; ainsi, faire la lessive ou le ménage, cuisiner, avoir une faible résistance à l'alcool, être tendre ou pacifique, etc.

\section{RÉSUMÉS}

Dans cet article, on a tenté, tout d'abord, de présenter l'ethnomusicologue chinois - ou du moins le personnage ainsi désigné - qui fréquente régulièrement le Xinjiang. Son travail et ses façons de faire sont décrits. Puis deux portraits-types ont été brossés, l'un est celui de l'ethnomusicologue ouïgour et l'autre, celui de son collègue han et pékinois. Par opposition, et c'est ce qui est développé au cours de la dernière partie, mon personnage ne pouvait être perçu que comme atypique. Or les principales caractéristiques que ma personne combinait devinrent autant d'atouts. Etre femme donnait la possibilité d'être aussi asexuée, la solitude appelait l'adoption, et ma nationalité attestait - si besoin était - de ma « non han-ité ».

In this article, we have firstly attempted to present the Chinese ethnomusicologist, or at least the person who calls himself thus, who regularly visits Xinjiang. His work and working methods are described. We then sketched two typical outline portraits, one being of the Uyghur ethnomusicologist and the other of his Han and Pekinese colleague. By contrast, and this is something developed in the last part of the article, my character may only be viewed as atypical and in fact the combination of different characteristics became something of an asset. Being a woman enabled one to be asexual, the isolation encouraged adoption and my nationality was proof, - if needs be - of my « non Hanity ».

\section{AUTEUR}

\section{SABINE TREBINJAC}

Sabine Trebinjac est docteur en ethnologie et chercheur au CNRS. Elle a vécu plusieurs années en République populaire de Chine en tant qu'étudiante (Université de Pékin et Conservatoire de musique), puis a passé une année sur le terrain dans la province du Xinjiang. Ses recherches sont consacrées à l'organisation de l'Etat chinois, qu'elle étudie par le biais des institutions musicales, et au traditionalisme d'Etat, avec ses conséquences musicales. De son séjour chez les Ouigour, elle 
a rapporté des enregistrements, qui ont été publiés par OCORA/AIMP, et sa thèse intitulée Musique ouïgoure et collectes musicales en Chine, qui sera publiée en trois volumes par la Société française d'ethnologie, Nanterre, 1995-1996. 